\title{
A high speed retro-reflective free space optics links with UAV
}

\author{
C. Quintana, Q. Wang Member, IEEE, D. Jakonis, O. Oberg, G. Erry, D. Platt Member, IEEE, Y. \\ Thueux, G. Faulkner, H. Chun, A. Gomez, M. Salter Member, IEEE, and D. O'Brien Member, IEEE
}

\begin{abstract}
In this work we report the design and implementation of a very high speed retro-reflective free space communication system between a ground station and a commercial unmanned aerial vehicle (UAV). The system uses a pixelated electro-absorption modulator (EAM) modulating retroreflector (MRR) to establish a data link operating at $500 \mathrm{Mbps}$ at a range of $560 \mathrm{~m}$ and a bit error rate (BER) of $7.6 \cdot 10^{-4}$. The MRR provides an effective aperture of $11 \mathrm{~mm}$ and full field of view (FFOV) of 6.4 ${ }^{\circ}$. To the best of our knowledge, this is the fastest demonstration of an outdoor link of this type. In this paper the design and implementation of the system is described, as well as results from experimental trials.
\end{abstract}

Index Terms - Free-space optical communications, quantum well devices, modulating retro-reflector, tracking, spatial light modulator.

\section{INTRODUCTION}

$\mathrm{R}$ adio-Frequency (RF) systems are the preferred technology to implement wireless communications links. Free space optics (FSO) can provide a high performance alternative, particularly in applications where RF spectrum is scarce and there are restrictions terminal size, weight and power ( $\mathrm{SWaP})$ [1]. FSOs systems also provide intrinsically more secure links than in RF due to the high directionality of optical beams [2].

Conventional symmetrical FSO systems typically make use of quite complex pointing and tracking (PTA) mechanisms to point the transmitting terminal towards the receiving terminal, and in many cases the receiving terminal to the transmitting.

There are certain applications with severe SWaP limitations (ie. small form Unmanned aerial vehicles (UAVs) or High Altitude Pseudo Satellites (HAPS)) where the use of traditional symmetrical FSO systems is impractical. RFSO (retroreflective free space optics) systems overcome this disadvantage. RFSO links use a high power unmodulated laser beam (hereafter also referred as the interrogating beam) launched from a ground station towards a modulating retroreflector (MRR) located in a mobile terminal. The MRR reflects a fraction of the incident optical radiation back to the ground station. The MRR also contains a modulator, typically a shutter, which modulates the returned beam with the data, which is to

Manuscript received April 29, 2021; revised June 20, 2021; accepted June 22, 2021. Date of publication $x$

This work was supported by the European Commission. H2020 Grant Agreement: 637595.

C. Quintana, G. Erry and Y. Thueux are with Airbus Operations Ltd, Building 07Y, Aerospace Avenue, Filton, Bristol, BS34 7PA UK (email: crisanto.quintana@airbus.com)

A. Gomez is with Microsoft Research Ltd, 21 Station Road, Cambridge, CB1 2FB, United Kingdom. be transmitted from the mobile station. The returned beam is detected by a receiver at the ground station.

The MRR can be implemented using a liquid crystal (LC), micro-electro-mechanical (MEMs) devices or Electro Absorption modulators (EAMs). LC MRRs typically consist of a LC optical shutter located in front of corner-cube retroreflector. Researchers at Lincoln Laboratory in Boston have reported high speed LCs with 30-50ns response times at $632 \mathrm{~nm}$ [3]. However, at the $1500 \mathrm{~nm}$ C-band wavelengths commonly used for free-space communications the viscosity of the required materials is increased which increases the device response time to the hundreds of micro-second regime [4]. MEMs based MRRs are traditionally implemented by using a MEMs deformable mirror as one facet of a hollow corner cube retro-reflector. They provide high contrast ratio (CR) and field of view (FOV) but the switching speed is limited to hundreds of $\mathrm{kHz}$ [5]. EAM based MRRs consist of a folded telecentric optical system that focuses the interrogating beam into an EAM modulator working in reflective mode. EAMs operate using the quantum-confined Stark effect (QCSE), which describes the effect of an external electric field upon the light absorption spectrum of a quantum well. Data links of 70 and $45 \mathrm{Mbps}$ have been demonstrated by the Naval Research Laboratory (NRL) in lab and outdoor scenarios [6-7].

In this paper, we report a demonstration data link of 500 Mbps between a flying unmanned aerial vehicle (UAV) and a ground station using a MRR, at a range of $560 \mathrm{~m}$. To the best of our knowledge, this is the fastest RFSO outdoor demonstration reported so far. This demonstrator makes use of a novel EAM based MRR that uses pixelation to reduce the EAM capacitance and increase the modulator bandwidth. The rest of the paper is organised as follows: Section 2 describes the system terminals design (ground station and MRR). Section 3 shows the link budget and experimental results obtained during the trials. Finally, conclusions and future work are presented in section 4.

\section{SYSTEM DESIGN}

The demonstrator consists of a ground station and a terminal mounted on an Unmanned Aerial Vehicle (UAV). The ground station is equipped with a high-power unmodulated laser

H. Chun is with Department of Information and Telecommunication Engineering, Incheon National University, Incheon, 22012, Korea

G. Faulkner, and D. O'Brien are with the Department of Engineering Science, University of Oxford, Oxford OX1 3PJ, U.K.

Q. Wang, D. Jakonis, O. Oberg, D. Platt and M. Salter are with RISE Research Institutes of Sweden AB, Electrum 236, 16440, Kistra, Sweden. (email: Qin.Wang@ri.se) 
(interrogator) and a high precision tracking system that directs the interrogator beam towards the UAV location. The UAV carries an MRR that captures a fraction of the interrogator beam, then modulates and reflects it back towards the ground station. The following subsections describe the ground station and MRR design.

\section{A. Ground station design}

Fig. 1 shows a picture of the ground station, which consists of coarse and fine-tracking modules working cooperatively. The coarse system uses a mechanical gimbal to rotate the body of the ground station, which contains the fine-tracking optical system. This gimbal (pan and tilt unit (PTU)) provides a large flexibility and wide range of operations (336 and $120^{\circ}$ in pan and tilt axis, respectively). The fine-tracking module offers a fast and high-precision pointing and operates over a range of $\pm 0.3^{\circ}$. This fine-tracking module is essential for the system to work, as a very precise alignment is required between both terminals. Additionally, it needs to cope with vibrations that may exist on the platform and also perturbation caused by the atmospheric turbulence effects, such as beam wander [8]. In the next section the coarse and fine-tracking system are described.

1) Coarse tracking system

The coarse tracking system is based on a mechanical PTU (FLIR D300) that holds the body of the ground station. The PTU is controlled using video signals captured by a visible CCD camera and a video tracker board (FLIR Vilga). A variable zoom lens (Computar H30Z1015) is also used to increase the precision of the video tracker at long distances. The FOV of the coarse tracking optical system can be adjusted from $\pm 9.1^{\circ}$ to $\pm 0.297^{\circ}$.

\section{2) Fine-tracking systems}

Figure 1 shows the fine-tracking module. This consists of a transmitter and a receiver optical system arranged in a bi-static configuration. This design improves the isolation between both optical chains but increases the mass of the system. Both transmitter and receiver optical chains are described in the following subsections.

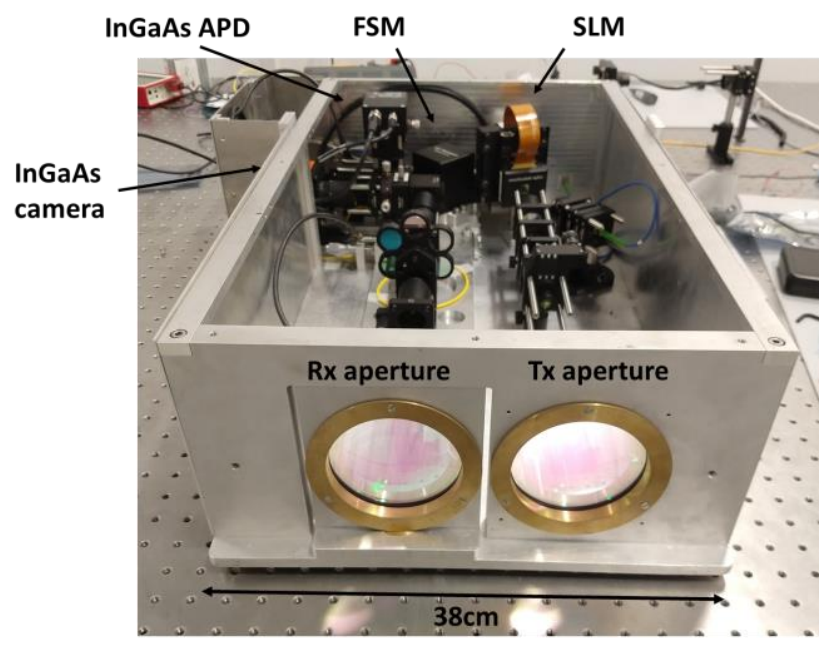

Fig. 1. Ground station fine-tracking optical system

a) Fine-tracking transmitter system

Fig. 2 shows the layout of the transmitter chain. A $1561 \mathrm{~nm}$ continuous wave (CW) fibre-coupled source, limited to $4 \mathrm{~W}$, is coupled to the system, and collimated using Lens 1 (Thorlabs AC254-045-C) before hitting a spatial light modulator (SLM) (Meadowlark HSP512-1550) arranged in an off-axis configuration. The SLM works in phase-only mode and can imprint phase differences up to $3 \pi$ with an 8-bit resolution. Holograms of $512 \times 512$ pixels can be written on the SLM. These consist of phase gratings to perform analogue beam steering up to $\pm 2.96^{\circ}$, as well as phase profiles to control beam divergence and high order aberration correction [9].

The light reflected off the SLM is expanded by a factor of 10 using a beam expander (Lens 4, Thorlabs AC127-019-C, and Lens 5, Thorlabs AL100200-C). This expansion also reduces the transmitter chain FOV to $\pm 0.296^{\circ}$. A $1: 1$ relay system (Lens 2 and Lens 3 ) is used to minimize aberrations in the optical path. The diameter of the beam at the transmitter aperture (Lens 5) is $79 \mathrm{~mm}$. This beam is classified as eye-safe (class 1M) by the BS EN60825-1:2014 standard as the irradiance does not exceed $1000 \mathrm{~W} \cdot \mathrm{m}^{-2}$.

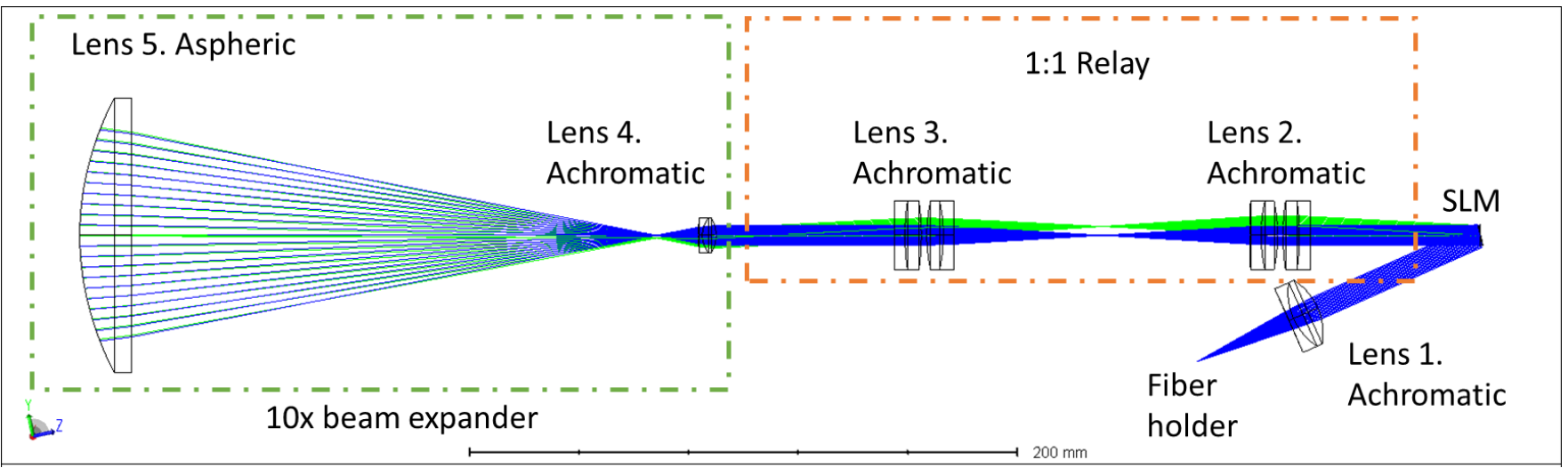

Fig. 2. Transmitter optical chain. 
b) Fine-tracking receiver system

Fig. 3 shows the design of the fine-tracking receiver chain. The light reflected from the MRR is captured at the ground station by a $79 \mathrm{~mm}$ aperture optical system. Lens 1 (Thorlabs AL100200-C), 2 (Thorlabs AL2018-C) and 3 (Edmund Optics 45826) form a 10x beam compressor. A field lens (Lens 2) was added to minimize aberrations introduced by the beam compressor. Lenses 2 and 3 are mounted on a motorized linear translation stage, which allows precise control of the collimation of the received light. An optical band-pass filter (Thorlabs FBH1550-12) was also introduced to reduce the background ambient light.

After a 1:1 relay system (Lens 4 and 5), a small fraction of the beam is sampled using a 10:90 beam splitter (BS1). The reflected light is sensed by an InGaAs camera (Bobcat Xenics 640) for tracking purposes. This sensor provides a pixel size of $20 \mu \mathrm{m}$ and an active area of $12.8 \times 10.2 \mathrm{~mm}$. Lens 5 (Edmund optics 67565) is a plano-convex lens with $90 \mathrm{~mm}$ focal length that sets the FOV of this arm to $\pm 0.3^{\circ}$.

In operation a focussed spot is formed on the camera sensor plane, with its position relative to the centre providing beam angle-of-arrival information. The captured frames on the camera are transferred to a graphical processing unit (GPU), where the position of the MRR is calculated using centroidfinding algorithms. Tracking errors up to $8 \mathrm{~cm}$ root-meansquared (RMS) at a range of $1.2 \mathrm{~km}$ have been reported using a similar system [10]. The calculated coordinates are then used to generate the holograms required to control the SLM located on the transmitter chain.

The transmitted beam after BS1 is corrected using a $1^{\text {st }}$ order adaptive optics (AO) module providing simple tip-tilt and finally focused (using Lens 7, Edmund Optics 47730) onto a high speed InGaAs photo-detector (Thorlabs APD430C). This photo-detector has an active area diameter of $200 \mu \mathrm{m}$ and a 3 $\mathrm{dB}$ bandwidth of $400 \mathrm{MHz}$. The AO system is based on a fast steering mirror (Newport FS-300), which has an aperture of 25 $\mathrm{mm}$ and a mechanical steering range of $\pm 1.5^{\circ}$. This device is controlled using a quad photo-detector (Thorlabs PDQ30C) and a closed-loop system control running at $600 \mathrm{~Hz}$. This simple AO system provides a robust stabilization of the received beam onto the InGaAs photo-detector and provides a FOV of this arm of $\pm 0.3^{\circ}$. Additionally, this system is also capable of compensating for a significant fraction of the aberrations $\left(1^{\text {st }}\right.$ order only) introduced by the atmospheric turbulence.

Although the system uses a photo-detector illuminated by the incoming beam directly it is designed to be compatible with a fibre coupled receiver. This solution would allow the use of erbium doped fibre amplifiers (EDFA) and ultra-high speed photo-detectors, which would increase both the sensitivity and bandwidth of the receiver chain.

\section{B. MRR design}

The MRR developed for the system is based on a cat's eye design, and Fig. 4 shows its architecture. It consists of a primary lens system that focuses the interrogating beam onto the EAM which operates in a reflective mode. This type of design increases the MRR aperture, which has a significant impact on the system link budget as the received power is proportional to fourth power of the MRR aperture [11]. The MRR also requires electronics to drive the EAM. These three subsystems are described below:

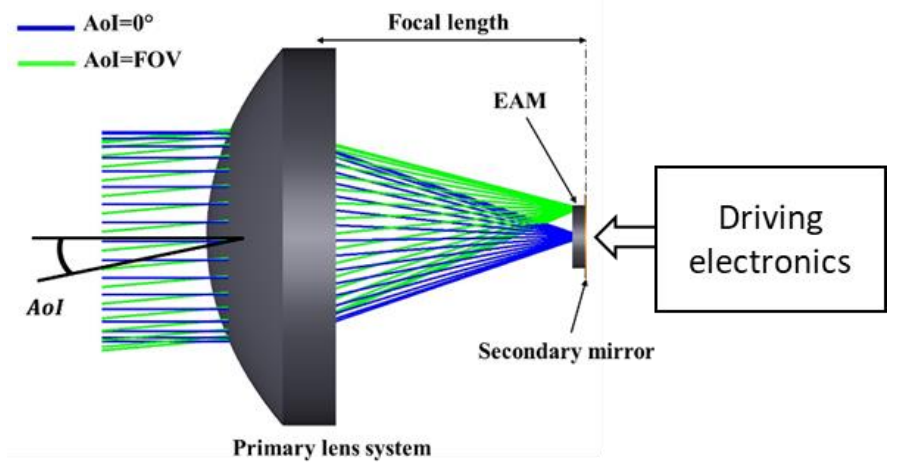

Fig. 4. MRR architecture. AoI refers to the angle of incidence.

\section{1) EAM design}

A surface-normal EAM optimised for C-band operation was designed and manufactured at RISE (Research Institutes of Sweden $A B$ ), one of the project partners. The design is based on 80 coupled InGaAs/InAlAs MQW embedded in an asymmetric Fabry-Perot (FP) cavity. This design leads to a $1.778 \mu \mathrm{m}$ thick modulator which provides a good CR with an acceptable insertion loss [12-13].

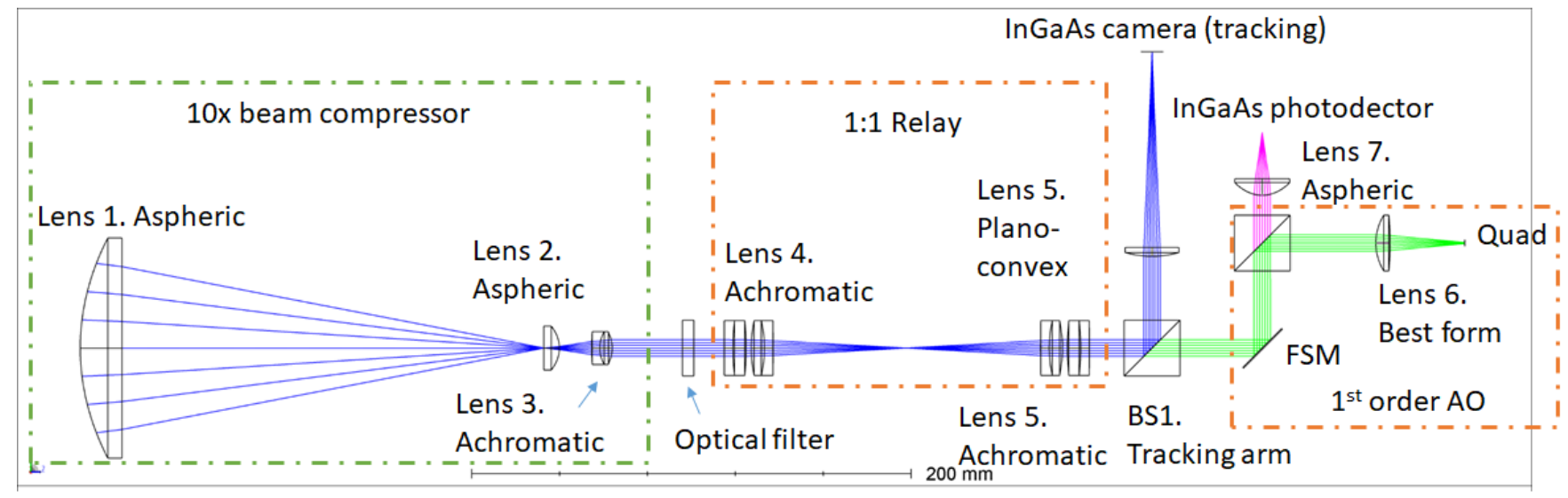

Fig. 3. Fine tracking receiver chain. 
The bandwidth of MQW modulators is inversely proportional to their active area, so a $6 \times 6$ array of modulators was designed, each modulator having the potential to modulate at rates of $1-2$ Gbps.

Fig. 5 shows the fabricated EAM. The device has a pixel size of the $250 \mu \mathrm{m} \times 250 \mu \mathrm{m}$ and a gap of $5 \mu \mathrm{m}$ to ensure sufficient isolation, leading to a $1.65 \times 1.65 \mathrm{~mm}$ device. Small wirebonding pads (50 $\mu \mathrm{m} \times 25 \mu \mathrm{m})$ were also added to connect the pixels to the driver electronics. Further details about the modulator are given in a previous publication [14].

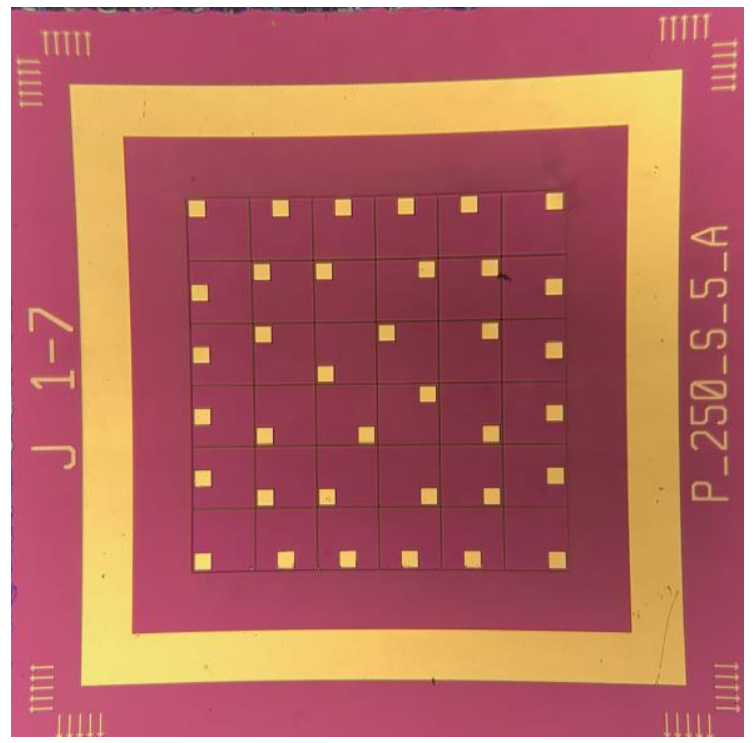

Fig. 5. Manufactured pixelated EAM.

\section{2) Driver electronics}

The driver electronics module acts as an interface between the data source and the MRR, and drives each pixel with a suitable voltage level. Low voltage differential signals (LVDS) provide binary data inputs to the MRR. The driver consists of an LVDS buffer that provides 8 independent outputs. Each output is pre-amplified using 8 dual output stages (16 driver outputs in total). Each line is connected to two pixels of the EAM. The 4 corner pixels are not in use as the light footprint on the EAM is circular. Driving 2 pixels in parallel reduces the MRR driver power consumption at the expense of halving the modulation bandwidth. With this configuration, the total system consumption is about $8 \mathrm{~W}$ and it could be reduced further by smart switching on and off of the drivers and pixels that are not in use. All the cathodes of the EAM pixels are connected to a common bias fixed to $4.6 \mathrm{~V}$. Fig. 6 a) shows the manufactured circuit, which includes the driver electronics and the EAM connected using bonding wires. Fig. 6 b) shows the electronics block diagram.

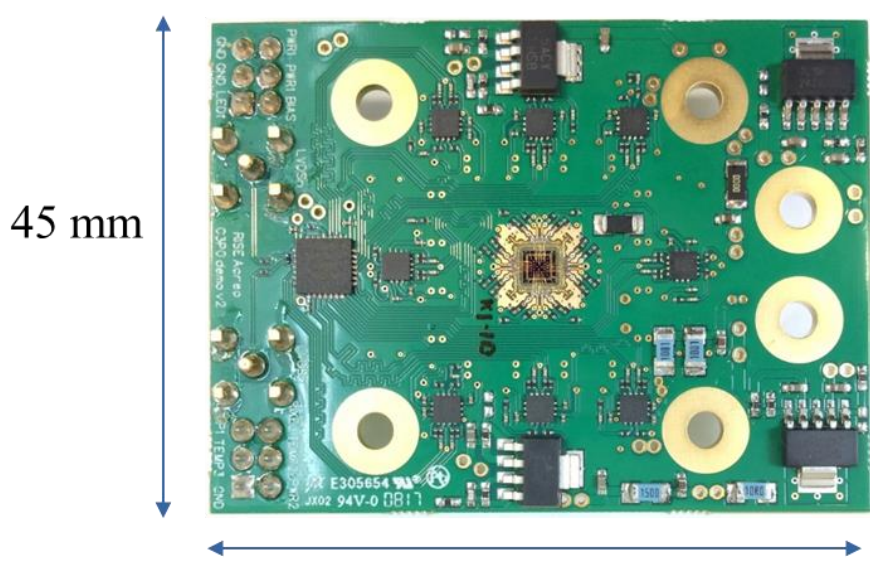

\section{$60 \mathrm{~mm}$}

A)

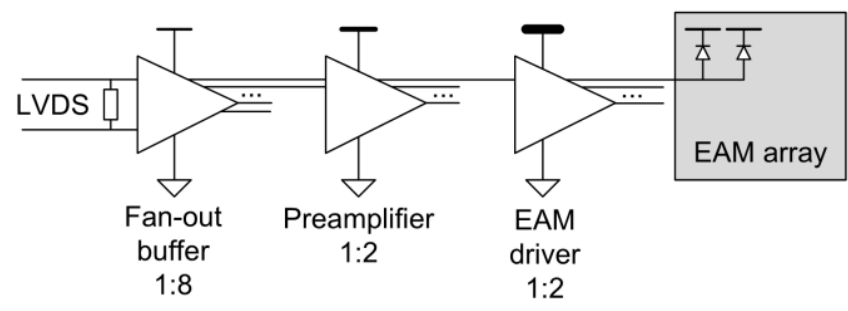

B)

Fig. 6. A) Developed PCB, which includes both EAM and driver electronics, B) Electronics block diagram.

\section{3) Optical system}

Fig. 7 shows the layout of the MRR optical design. It consists of a 2-lens optical system that provides an effective aperture of $11 \mathrm{~mm}$ and a FOV of $\pm 3.2^{\circ}$. Lens 1 is an aspheric plano-convex (Thorlabs AL1225-C) with $12.5 \mathrm{~mm}$ aperture and $25 \mathrm{~mm}$ focal length. Lens 2 is an achromatic doublet (Edmund optics \#65435) with $9 \mathrm{~mm}$ aperture and a focal length of $12 \mathrm{~mm}$. The distance between them was set to $8.75 \mathrm{~mm}$ to minimize spherical aberrations. A precise axial location of the EAM in the focal plane of the optical system is essential to achieve a diffraction-limited returned beam after reflection off the EAM. Additionally, the EAM must be placed normal to the optical axis in order to avoid any angular offset between the interrogator and the returned beam. Accurate mounting stages were used to achieve these requirements.

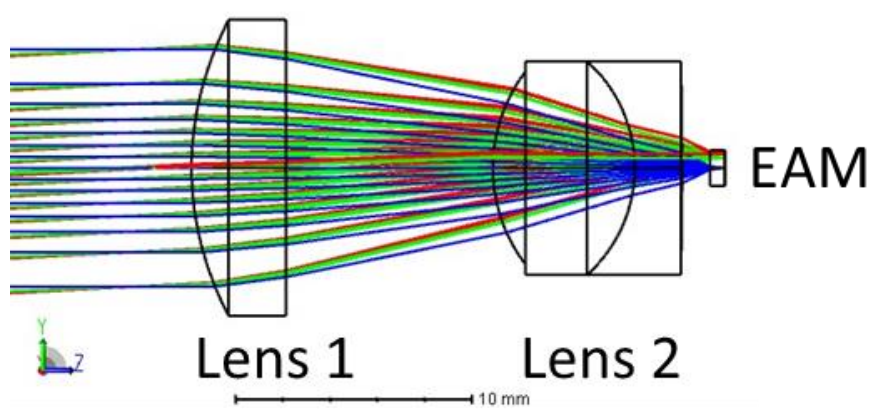

Fig. 7. Cat's eye optical design

The aberrations introduced by this cat's eye optical system were modelled in Zemax. Fig. 8 shows the diffraction encircled energy for different incidence angles. An ideal optical system 
would provide an $\mathrm{M}^{2}=1$, and values of 1.3 have been estimated for incidence angles up to $2^{\circ}$ and 3.4 for the maximum incidence angle $\left(3.2^{\circ}\right)$.

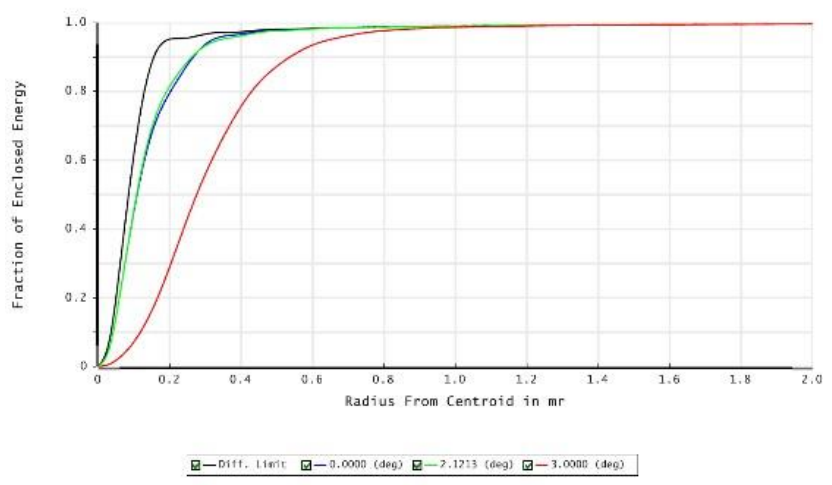

Fig. 8. Cat's eye diffraction encircled energy

This optical system has been designed for an operating temperature of $20^{\circ} \mathrm{C}$. Environmental simulations run on Zemax determined that the $\mathrm{M}^{2}$ of the optical system increases to 1.7 for incidence angles up to $2^{\circ}$ at extreme temperatures of 0 and 50 ${ }^{\circ} \mathrm{C}$.

\section{RESULTS}

In this section we present the trials setup, link budget calculations and the results obtained on the different tests. These trials consisted of static tests (MRR set up on a tripod) at a range of $250 \mathrm{~m}$ and dynamic tests with the MRR attached to a flying UAV located $560 \mathrm{~m}$ away from the ground station. The trials were performed in the UK the first week of September. The temperature was about $18^{\circ} \mathrm{C}$ with cloudy weather conditions.

\section{A. Link budget}

TABLE I

PARAMETERS CONSIDERED TO CALCULATE THE LINK BUDGET.

\begin{tabular}{cc}
\hline \hline Parameter & Value \\
\hline Laser power $\left(\mathrm{P}_{\mathrm{tx}}\right)$ & $4 \mathrm{~W}(36 \mathrm{dBm})$ \\
Transmitter loses $\left(\mathrm{L}_{\mathrm{tx}}\right)$ & $1.67 \mathrm{~dB}$ \\
Interrogator beam diameter & $8 \mathrm{~cm}$ \\
Transmitter $\mathrm{M}^{2}$ & 1.1 (beam divergence 18 \\
MRR M ${ }^{2}$ & $\mu$ rads) \\
Atmospheric absorption & 1.3 \\
loses $\left(\mathrm{L}_{\mathrm{Att} \text { abs }}\right)$ & $0.4 \mathrm{~dB} / \mathrm{km}$ \\
Link range & $560 \mathrm{~m}$ \\
Bi-static loses $\left(\mathrm{L}_{\mathrm{Bi}-\mathrm{static}}\right)$ & $13.7 \mathrm{~dB}$ \\
MRR aperture & $11 \mathrm{~mm}$ \\
Receiver aperture diameter & $8 \mathrm{~cm}$ \\
EAM insertion loses & $11.5 \mathrm{~dB}$ \\
$\left(\mathrm{~L}_{\mathrm{MRR}}\right)$ & $9.5 \mathrm{~dB}$ \\
Data receiver loses $\left(\mathrm{L}_{\mathrm{tx}}\right)$ & $-37 \mathrm{dBm}$ \\
Data receiver sensitivity &
\end{tabular}

Table 1 shows a summary of the system parameters. The sensitivity of the data receiver photo-detectors was measured in the lab and it was considered as the optical power at which the electrical output of the photo-detector presents a signal-to-noise ratio of $10 \mathrm{~dB}$. A value of $-37 \mathrm{dBm}$ was obtained. A BER lower than $10^{-7}$ was measured under these conditions.

The link budget has been calculated using equation 1 :

$$
\begin{aligned}
P_{r x}= & P_{T x} \cdot L_{T x} \cdot G_{T x} \cdot \mathrm{L}_{\mathrm{Att}}{ }_{\mathrm{abs}} \cdot L_{M R R} \\
& L_{R x} \cdot \mathrm{L}_{\mathrm{Att} \_\mathrm{abs}} \cdot G_{M R R} \cdot L_{B i-s t a t i c}
\end{aligned}
$$

Where $\mathrm{P}_{\mathrm{Rx}}$ is the average received optical power on the data photo-detector, $\mathrm{P}_{\mathrm{Tx}}$ represents the optical power of the interrogator beam, $\mathrm{L}_{\mathrm{Tx}}$ and $\mathrm{L}_{\mathrm{Rx}}$ are the transmitter and receiver loses. $\mathrm{G}_{\mathrm{Tx}}$ are the geometrical loses of the interrogator beam at the MRR and $\mathrm{G}_{\mathrm{MRR}}$ are the geometrical loses of the retroreflected beam at the ground station considering a monostatic configuration. $\mathrm{L}_{\mathrm{Bi} \text {-static }}$ refers to the increase of $\mathrm{G}_{\mathrm{MRR}}$ loses caused by the use of a bi-static configuration. These loses are introduced by the fact that the returned beam is centered on the ground station transmitter aperture and not on the receiver one. $\mathrm{L}_{\text {Att_abs }}$ refers to the atmospheric absorption loses and $\mathrm{L}_{\mathrm{MRR}}$ are the insertion loses of the EAM.

$\mathrm{G}_{\mathrm{Tx}}$ has been calculated as the ratio of the squared beam width of the interrogator beam at the MRR and diameter of the aperture. The beam width at the MRR was calculated using the beam diameter at the source $(8 \mathrm{~cm})$, the Rayleigh length, the $\mathrm{M}^{2}$ and the link range. The same approach was used to calculate the beam diameter of the returned beam at the ground station and then the $\mathrm{G}_{\mathrm{MMR}}$.

The bi-static configuration of the ground station has a negative impact on the link budget calculation at short range. Numerical simulations were performed in Matlab to calculate the loses introduced by the offset between the receiver and the transmitter aperture. This parameter contributes with $13.7 \mathrm{~dB}$ loses at a range of $560 \mathrm{~m}$. At this range, this parameter is significantly larger than the geometrical loses that would be on a mono-static system $\left(\mathrm{G}_{\mathrm{MRR}}=2.8 \mathrm{~dB}\right)$.

For this particular case shown in table I, the average received power expected at the receiver photo-detector $\left(\mathrm{P}_{\mathrm{rx}}\right)$ is about $19.59 \mathrm{dBm}$.

\section{B. Static tests}

The static tests were performed with the MRR set up on tripod $250 \mathrm{~m}$ away from the ground station. A $1561 \mathrm{~nm} \mathrm{CW}$ interrogator beam with a power of $20 \mathrm{dBm}$ was transmitted from the ground station towards the MRR. The MRR was modulated using a non-return-to-zero (NRZ) on-off-keying (OOK) scheme using a 500Mbps pseudo-random binary sequence (PRBS) generated on a Xilinx ML507 evaluation board.

At the ground station, the received signal output from the photo-detector was sampled with a high speed oscilloscope for later post-processing. Adaptive thresholding techniques were used to remove the low frequency intensity variation introduced by the atmospheric turbulence. Fig. 9 shows the eye diagram of the captured signal after this processing. It shows a limited jitter on the received signal as well as an acceptable SNR that would allow successful thresholding and sampling. The average received optical power in this experiment was $-19.21 \mathrm{dBm}$. A decision feedback equalizer (DFE) was also used to remove some of the inter-symbol interference added by the MRR driver electronics. This equalizer uses 35 and 25 feed-forward and feedback filter taps, respectively, and a sequence of 350 bits 
was employed to train the filters. A resulting bit error rate (BER) of $1.22 \cdot 10^{-4}$ was measured over a period of 6 secs. Most of these errors are caused by the scintillation effect introduced by the atmospheric turbulence. However, this BER is sufficiently low that an error-free link can be achieved by using forward error correction (FEC) codes and interleavers [15-16].

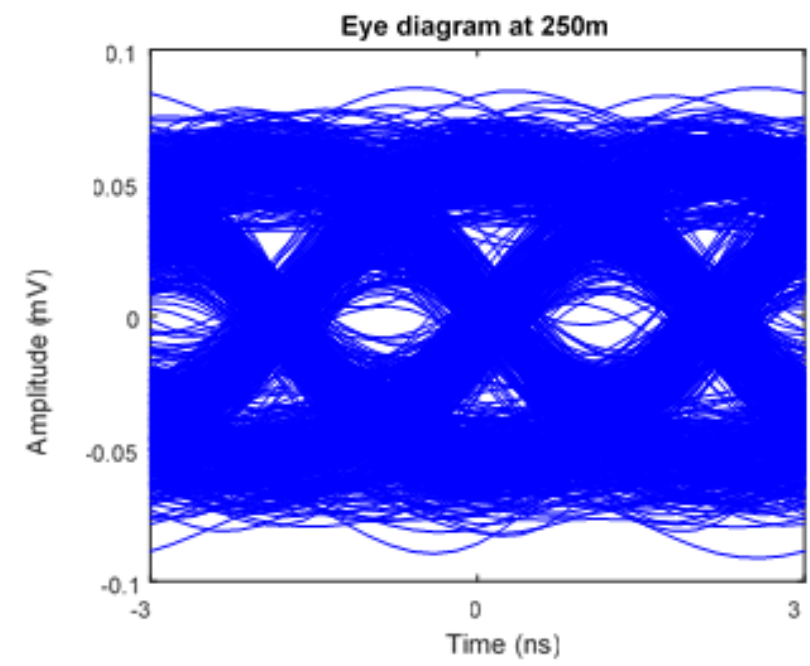

Fig. 9. Eye diagram captured for a link range of $250 \mathrm{~m}$ (static).

\section{Dynamic tests}

The dynamic test was performed with the MRR attached to a UAV. An octocopter (Cinestar 8's Mikrokopter) was used on this test. Fig. 10 shows the setup before taking off. In this demonstration the link range was $560 \mathrm{~m}$ and the power of the interrogator beam was increased to $36 \mathrm{dBm}$. This increase in the transmitted power was required to compensate for the $\mathrm{L}_{\mathrm{bi}}$ static loses described in section III.A.

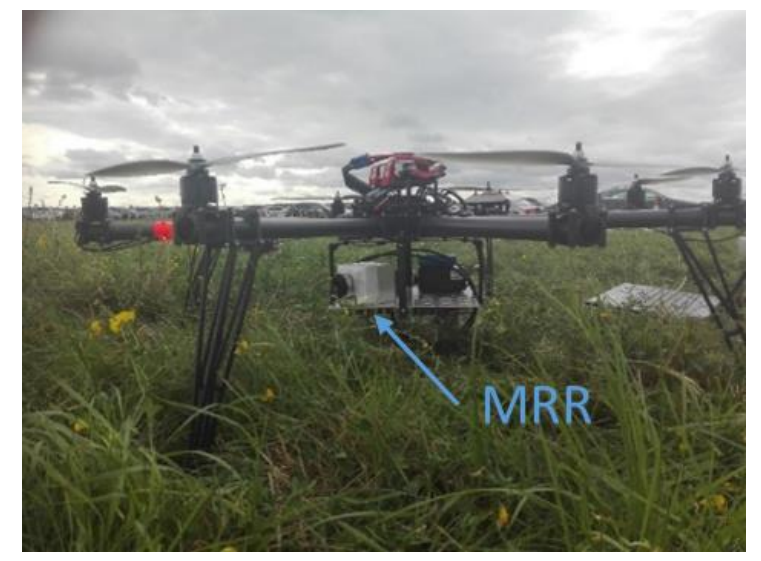

Fig. 10. Dynamic tests with the MRR attached to the UAV.

Once the coarse tracking of the ground station points the optical terminal towards the MRR, the fine-tracking module starts the acquisition stage which is required to locate the MRR. During this stage, the SLM is used to increase the beam divergence and perform raster scanning, and the increase in divergence of the beam allows the field of view to be covered rapidly. Once a strong reflection from the MRR is sensed on the InGaAs tracking camera located at the ground station, the interrogator beam is returned to collimation and the tracking stage is enabled. This process is fully automated.
Figure 11 shows the transmitted and received data. In this case, an average optical power of $-17.96 \mathrm{dBm}$ was measured on the data photo-detector. A value of $-19.59 \mathrm{dBm}$ was estimated in the link budget simulation shown in III.A. This simulated value is $1.5 \mathrm{~dB}$ lower than the one measured on the experiment. This disagreement is caused by a slight misalignment on the MRR optics, which increased the divergence of the returned beam and then reduced the bi-static loss. An MRR $\mathrm{M}^{2}$ of 1.6 would provide a much closer match between the link budget and the trials measurement.

Similar to the static tests, the received signal was postprocessed using adaptive thresholding and DFE. A BER of 7.6. $10^{-4}$ was measured during burst of data of a few seconds. The stability of the link was compromised by the heading accuracy of the UAV during manoeuvring, the limited FOV of the MRR and the windy conditions on the trials' day. However, similarly to static test, this BER value can be handled by FEC codes and provide an error-free link.

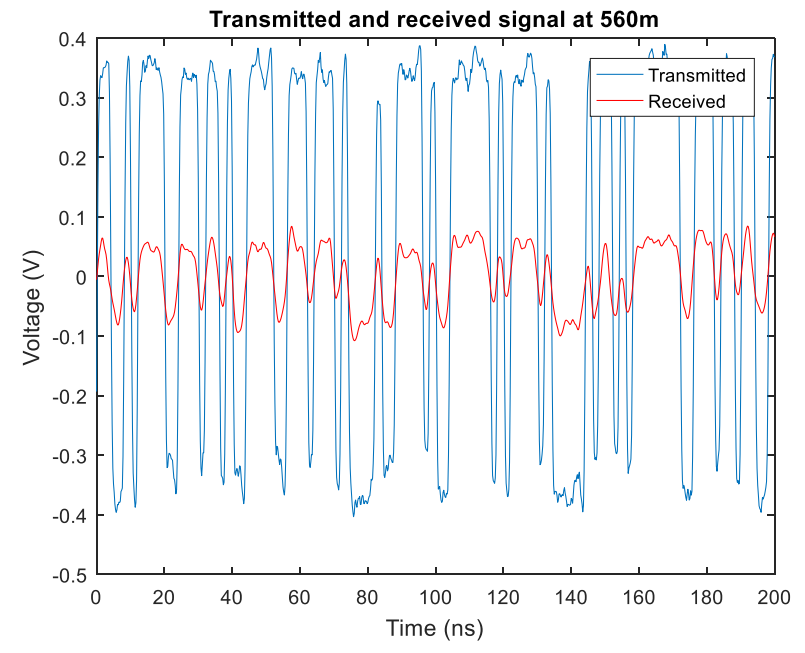

Fig. 11. Transmitted and received signal during flight at $560 \mathrm{~m}$.

\section{CONCLUSION}

MRR based communication is well-suited to UAVs and similar platforms where SWaP are highly constrained. In this work we report an operating high data-rate link that demonstrates this concept. A BER of $7.6 \cdot 10^{-4}$ was measured during flight tests for data link operating at $500 \mathrm{Mbps}$ at a range of $560 \mathrm{~m}$. To the best of our knowledge, this is the fastest demonstration of an outdoors RFSO data link.

Future work includes the improvement of the MRR driving electronics, which should increase the link data rate to at least 1 Gbps. In the longer term, increasing the number of pixels would increase MRR FOV, which would provide more robustness to the data link against challenging weather conditions. At the ground station, further tests are required using a fibre based detector. Coupling light into a single mode fibre would allow the use of EDFA amplifiers and fibre detector, which would increase the bandwidth and the sensitivity of the receiver system.

\section{REFERENCES}


[1] H. Kaushal and G. Kaddoum, "Optical Communication in Space: Challenges and Mitigation Techniques," in IEEE Communications Surveys \& Tutorials, vol. 19, no. 1, pp. 57-96, 2017.

[2] Arun K.Majumdar, "Advanced Free Space Optics (FSO) A Systems Approach", Springer Series in Optical Sciences 186, 2015.

[3] LC M. W. Geis, T. M. Lyszczarz and R. Osgood, "30 to 50 ns liquidcrystal optical switches," Optics Express, vol. 18, no. 18, pp. 1888618893, 2010.

[4] B.E.A. Saleh and M.C. Teich, "Fundamentals of Photonics", Wiley, Hoboken, NJ, 1991, pp. 696-736,

[5] Jason B. Stewart, David Freedman, Steven Cornelissen, Mark N. Horenstein, Peter Woskov, Jonathan Tang, "Low power MEMS modulating retroreflectors for optical communication," Proc. SPIE 7595, MEMS Adaptive Optics IV, 759505 (18 February 2010);

[6] W.S. Rabinovich, P.G. Goetz, R. Mahon, L. Swingen, J. Murphy, G.C. Gilbreath, S. Binari,E. Waluschka, "Performance of Cat's eye modulating retro-reflectors for free-space optical communications" Proc. SPIE 5550, pp. 104-114, 2004.

[7] W.S. Rabinovich, P.G. Goetz, R. Mahon, L. Swingen, J. Murphy, M. Ferraro, H. Ray Burris, Jr., C.I. Moore, M. Suite, G. Charmaine Gilbreath, S. Binari, D. Klotzkin, "45-Mbit/s Cat's eyemodulating retro-reflectors." Opt. Eng. Vol. 46(10), 104001, 2007.

[8] H. Kaushal et al., "Experimental Study on Beam Wander Under Varying Atmospheric Turbulence Conditions," in IEEE Photonics Technology Letters, vol. 23, no. 22, pp. 1691-1693, Nov.15, 2011

[9] C. Quintana, G. Erry, A. Gomez, Y. Thueux, G. E. Faulkner, and D. C. O'Brien, "Design of a holographic tracking module for long-range retroreflector free-space systems," Applied Optics, vol. 55, pp. 7173$7178,2016$.

[10] C. Quintana, G. Erry, A. Gomez, Y. Thueux, G. Faulkner and D. O'Brien, "Novel non-mechanical fine-tracking module for retroreflective free space optics" SPIE 9248, Unmanned/Unattended Sensors and Sensor Networks X, vol. 92480S, 2014.

[11] G. C. Gilbreath, W.S. Rabinovich, T.J. Meehan, M.J. Vilcheck, R. Mahon et al., "Large-aperture multiple quantum well modulating retroreflector for free-space optical data transfer on unmanned aerial vehicles", SPIE Opt. Eng.,vol. 40(7), pp. 1348-1356, 2001.

[12] T. H. Stievater, W. S. Rabinovich, P. G. Goetz, R. Mahon and S. C. Binari, "A surface-normal coupled-quantum-well modulator at $1.55 \mu \mathrm{m}$ " in IEEE Photonics Technology Letters, vol. 16, no. 9, pp. 2036-2038, Sept. 2004

[13] Q. Wang, B. Noharet, S. Junique, S. Almqvist, D. Agren and J. Y. Andersson, "1550 nm transmissive/reflective surface-normal electroabsorption modulator arrays", IEEE Elec. Lett., vol. 42 (1), pp. 47 63, 2006.

[14] C. Quintana, C. Quintana, Q. Wang, D. Jakonis, X. Piao, G. Erry, D. Platt, Y. Thueux, A. Gomez, G. Faulkner, H. Chun, M. Salter, and D. O'Brien; "High Speed Electro-Absorption Modulator for Long Range Retroreflective Free Space Optics,"; IEEE Photonics Technology Letters, vol. 29, no. 9, pp. 707-710, 2017.

[15] Greco, Joseph A. "Design of the high-speed framing, FEC, and interleaving hardware used in a $5.4 \mathrm{~km}$ free-space optical communication experiment." Free-Space Laser Communications IX. Ed. Arun K. Majumdar \& Christopher C. Davis. San Diego, CA, USA: SPIE, 2009

[16] A. Alvarado, E. Agrell, D. Lavery, R. Maher and P. Bayvel, "Replacing the Soft-Decision FEC Limit Paradigm in the Design of Optical Communication Systems," J. Lightwave Technol., vol. 33, pp. 4338-4352, 2015. 